\title{
Geostrophic adjustment in a closed basin
}

\author{
E. R. J OH N O $\mathrm{N}^{1}$ AND R. H. J. GR IMSHA W \\ ${ }^{1}$ Department of Mathematics, University College London, Gower Street, \\ London WC1E 6BT, U.K. \\ ${ }^{2}$ Department of Mathematical Sciences, Loughborough University, U.K.
}

(Received 1 May 2013)

\begin{abstract}
We consider geostrophic adjustment of a density stratified fluid in a basin of constant depth on an $f$-plane in the context of linearized theory. For a single vertical mode, the equations are equivalent to those for a linearized shallow water theory for a homogeneous fluid. These can be solved explicitly for general initial conditions in a circular domain. When the Rossby radius is much smaller than the basin radius, appropriate for the baroclinic modes, we find that the interior adjusted solution is close to that of the initial state, except for small-amplitude trapped Poincaré waves, while Kelvin waves propagate around the boundary, carrying the initial height field without change of form. This interpretation allows us to extend the results to domains of arbitrary shape, containing interior islands and with boundary gaps. In multiply-connected domains the average surface elevation on the solid boundaries is determined as part of the solution by an application of Kelvin's circulation theorem.
\end{abstract}

\section{Introduction}

Geostrophic adjustment occurs in a rotating fluid when an an initial state relaxes to a steady state in geostrophic balance. In the linearized theory for open systems, the adjustment typically occur by the generation of the waves allowed by the system, which propagate to infinity, and leave a steady state determined by geostrophic equilibrium and the conservation of potential vorticity, see for instance Gill (1982) amongst many works on this topic. For instance, in a long shallow channel filled with a fluid of constant density, the adjustment takes place through Poincaré and Kelvin waves, see Gill (1976). However, in a closed system, the adjustment takes a different form since the generated waves cannot escape to infinity, and hence the adjusted state does not become steady, and consists of both waves and the geostrophically balanced steady state. This situation arises for instance in lakes, where due to the density stratification, the response to temporally localised wind forcing is typically the generation of internal Poincaré and Kelvin waves, together with a steady component, see for instance Stocker \& Imberger (2003), Wake et al. (2004, 2005, 2007) and Gomez-Giraldo et al. (2006) for a combination of theory, numerical simulations, observations and experiments.

This present study is motivated by the recent numerical and theoretical study by Luneva et al. (2012) on geostrophic adjustment in the Arctic Ocean. They considered a homogeneous fluid in a circular basin, with idealised topography, and showed that after the Poincaré and Kelvin waves have been damped by friction, the flow adjusts to a quasi-steady state. Here we consider the adjustment of a density-stratified fluid in a constant-depth basin allowing for islands within the basin (and, later, gaps in the basin boundary). In this case, the governing equations for a single vertical mode are equivalent 


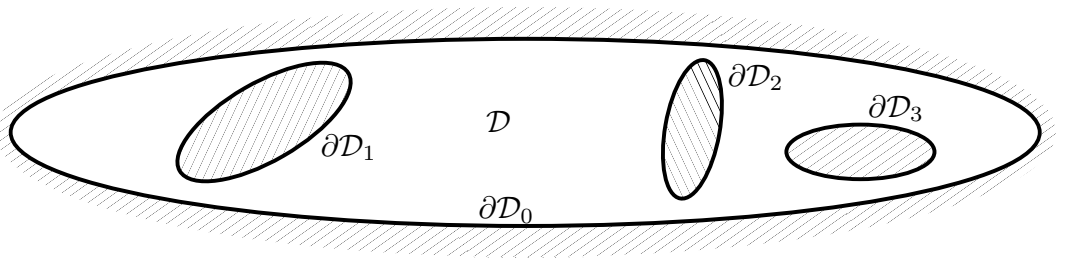

FiguRe 1 . The basin $\mathcal{D}$ with outer boundary $\partial \mathcal{D}_{0}$ and possible islands with boundaries $\partial \mathcal{D}_{i}$ $(i=1,2, \ldots)$.

to the linearized shallow water equations for a homogeneous fluid of constant depth on an $f$-plane. This latter case was discussed by Stocker \& Imberger (2003) for a linear initial tilt of the free surface, and as expected the solution consisted of Poincaré and Kelvin waves, and a steady geostrophic component. Here, we consider a range of initial states, and put a special emphasis on the limit when the Rossby radius is much smaller than the basin radius. Note that for the baroclinic vertical modes, this is the expected normal situation. In this case we will show that the solution is dominated by the steady geostrophic component, and boundary-trapped Kelvin waves.

In $\S 2$ we formulate the problem and obtain the governing equations for the unique steady geostrophic component. Then in $\S 3$ we take the explicit example of a circular basin, revisit the free waves first discussed by Lamb (1932), and formally present the full solution as a Laplace transform. It transpires that it is simpler to solve the full problem numerically, and these results are presented in $\S 3.3$. In $\S 4$ we return to the considering domains of arbitrary shape with interior islands and boundary gaps and show how our results apply in the limit when the Rossby radius is much smaller than typical geometric dimensions. We conclude in $\S 6$.

\section{Formulation}

We consider an incompressible, inviscid density stratified fluid with basic density $\rho_{0}(z)$ bounded above by a free surface at $z=\eta$ and below by a rigid boundary at $z=-H$, contained in a basin $\mathcal{D}$, with outer vertical boundary $\partial \mathcal{D}_{0}$, possibly containing islands also with vertical boundaries $\partial \mathcal{D}_{i}(i=1,2, \ldots)$ (Figure 1$)$ Then the linearized long-wave equations on an $f$-plane are, in conventional notation,

$$
\begin{aligned}
u_{x}+u_{y}+w_{z} & =0, \\
\rho_{0}\left(u_{t}-f v\right)+p_{x} & =0, \\
\rho_{0}\left(v_{t}+f u\right)+p_{y} & =0, \\
p_{z}+g \rho & =0, \\
\rho_{t}+\rho_{0 z} w & =0 .
\end{aligned}
$$

Here $p, \rho$ are the perturbation pressure and density respectively. The boundary conditions are

$$
\begin{aligned}
\zeta_{t}-w & =0, \quad z=0, \\
p-g \rho_{0} \zeta & =0 \quad z=0, \\
w & =0, \quad z=-H \\
\boldsymbol{u} . \hat{\boldsymbol{n}} & =0, \quad \text { on } \partial \mathcal{D},
\end{aligned}
$$

where $\partial \mathcal{D}=\partial \mathcal{D}_{0} \cup \partial \mathcal{D}_{1} \cup \ldots$, and, for definiteness, $\hat{\boldsymbol{n}}$ is the unit inward normal for $\mathcal{D}$. It is now useful to introduce the vertical particle displacement $\eta$. In the linearized theory 
this is defined by

$$
\eta_{t}=w .
$$

Then note that $\eta(z=0)=\zeta$ and the density equation (2.5) integrates to

$$
\rho=-\rho_{0 z} \eta
$$

A solution of this system of equation and boundary conditions can now be sought using separation of variables, see Gill (1982) for instance. That is, we write

$$
\eta=\tilde{\eta}(x, y, t) \phi(z), \quad(u, v)=(\tilde{u}, \tilde{v})(x, y, t) h \phi_{z}(z), \quad p=\tilde{\eta}(x, y, t) c^{2} \rho_{0}(z) \phi_{z}(z) .
$$

Here $c^{2}, h$ are separation constants which will be specified below. Then the modal function $\phi(z)$ satisfies the boundary value problem

$$
\begin{aligned}
& c^{2}\left(\rho_{0} \phi_{z}\right)_{z}-g \rho_{0 z} \phi=0, \quad-H<z<0, \\
& c^{2} \phi_{z}=g \phi, \quad z=0, \quad \text { and } \phi=0, \quad z=-H .
\end{aligned}
$$

Here $c$ is the linear long wave speed. In general, there are infinitely many solutions, which can be ordered by an index $m$, so that $\phi_{0}, c_{0}$ is the barotropic mode, and $\phi_{m}, c_{m}$, $(m=1,2, \ldots)$ are the baroclinic modes, ordered so that the $c_{m}$ decrease as $m$ increases. We fix attention on just one mode, and then find that

$$
\begin{aligned}
\tilde{\eta}_{t}+h\left(\tilde{u}_{x}+\tilde{v}_{y}\right) & =0, \\
h\left(\tilde{u}_{t}-f \tilde{v}\right)+c^{2} \tilde{\eta}_{x} & =0, \\
h\left(\tilde{v}_{t}+f \tilde{u}\right)+c^{2} \tilde{\eta}_{y} & =0,
\end{aligned}
$$

This system can now be recognised as the linearized shallow water equations with equivalent depth $h$ defined by setting $c^{2}=g h$. We remove the ${ }^{\sim}$ from $\eta, u, v$, and note that $(2.16,2.17)$ separate to give,

$$
\begin{aligned}
& u_{t t}+f^{2} u+g \eta_{x t}+g f \eta_{y}=0 \\
& v_{t t}+f^{2} v+g \eta_{y t}-g f \eta_{x}=0
\end{aligned}
$$

and so (2.9) becomes

$$
\eta_{n t}-f \eta_{s}=0, \quad \text { on } \partial \mathcal{D},
$$

where $s$ is a displacement around each $\partial \mathcal{D}_{i}$ such that $(\hat{\boldsymbol{z}}, \hat{\boldsymbol{n}}, \hat{\boldsymbol{s}})$ forms a right-handed system. This definition reflects the fact that Kelvin waves cycle anti-clockwise around the inside of the outer boundary $\partial \mathcal{D}_{0}$ but clockwise around the island boundaries $\partial \mathcal{D}_{1}, \partial \mathcal{D}_{2}, \ldots$

These equations are to be solved for an unbalanced density distribution released from rest,i.e.

$$
\eta=\eta_{I}(x, y), u=v=0, \quad \text { at } \quad t=0 .
$$

Equations $(2.15,2.16,2.17)$ give the potential vorticity equation

$$
v_{x t}-u_{y t}-(f / h) \eta_{t}=0
$$

which integrates to

$$
v_{x}-u_{y}-f \eta / h=-(f / h) \eta_{I} .
$$

It is thus possible, as in unbounded flow, to proceed directly to the unique steady solution $\eta_{S}(x, y)$ consistent with the initial conditions. In steady flow equations $(2.18,2.19)$ give the geostrophic relations

$$
u=-(g / f) \eta_{y}, \quad v=-(g / f) \eta_{x}
$$


and (2.23) becomes the inhomogeneous modified Helmholtz equation

$$
\nabla^{2} \eta-K^{2} \eta=-K^{2} \eta_{I}
$$

where horizontal distances have been scaled on $L$, a typical basin scale, and $K=f L / c$ is a Kelvin number measuring the basin scale in Rossby radii and also giving the number of inertial periods required for a Kelvin wave to cycle the basin. Note that $a=1 / K$ is the Rossby radius non-dimensionalised on the basin scale. Equation (2.25) is to be solved subject to the boundary conditions, from (2.20), that

$$
\eta=\alpha_{i}, \quad \text { on } \partial \mathcal{D}_{i}, \quad(i=0,1,2, \ldots),
$$

where the constants $\alpha_{i}$ give the time-average interface displacements at the boundaries and remain to be determined. When no island is present, $\alpha_{0}$ follows immediately from the conservation of mass,

$$
\int_{\mathcal{D}} \eta=M=\int_{\mathcal{D}} \eta_{I}
$$

for $M$ a constant determined by the initial conditions. When islands are present condition (2.20) can first be integrated around each island to give

$$
\frac{\mathrm{d}}{\mathrm{d} t} \oint \frac{\partial \eta}{\partial n} \mathrm{~d} s=0, \quad \text { on } \partial \mathcal{D}_{i}, \quad(i=0,1,2, \ldots),
$$

showing the circulation is constant around each island throughout the adjustment (Kelvin's theorem, as used in Lin (1941) for irrotational flow and by Rhines (1969), Johnson (1989)for rotating flow). Thus the steady solution must also satisfy the conditions

$$
\oint \frac{\partial \eta}{\partial n} \mathrm{~d} s=\oint \frac{\partial \eta_{I}}{\partial n} \mathrm{~d} s=\Gamma_{i} \text { (say), } \quad \text { on } \partial \mathcal{D}_{i}, \quad(i=0,1,2, \ldots),
$$

and these additional conditions determine the constants $\alpha_{i}$. The problem for $\eta_{S}$ is nonstandard and is solved most directly both analytically (as in Johnson (1989)) and numerically by exploiting the linearity of (2.25) first solving (2.25) subject to

$$
\eta=0 \quad \text { on } \partial \mathcal{D}_{i}, \quad(i=0,1,2, \ldots),
$$

to obtain $\eta_{S 0}$ (say) and then solving,for each $j$, the homogeneous form of (2.25) (with $\left.\eta_{I}=0\right)$ subject to

$$
\eta=1 \quad \text { on } \partial \mathcal{D}_{j}, \quad \eta=0 \quad \text { on } \partial \mathcal{D}_{i}, \quad(i \neq j), \quad(j=0,1,2, \ldots),
$$

to obtain solutions $\eta^{(j)}$ with island circulations

$$
G_{i j}=\oint_{\partial \mathcal{D}_{i}} \frac{\partial \eta^{(j)}}{\partial n} \mathrm{~d} s .
$$

The steady surface displacements at the islands are then given by the matrix equation

$$
\mathbf{G} \boldsymbol{\alpha}=\boldsymbol{\Gamma},
$$

for $\mathbf{G}=\left\{G_{i j}\right\}, \boldsymbol{\alpha}=\left\{\alpha_{j}\right\}, \boldsymbol{\Gamma}=\left\{\hat{\Gamma}_{i}\right\}$, where

$$
\hat{\Gamma}_{i}=\Gamma_{i}-\oint_{\partial \mathcal{D}_{i}} \frac{\partial \eta_{S 0}}{\partial n} \mathrm{~d} s .
$$

and the full interface displacement is given by

$$
\eta_{S}=\eta_{S 0}+\sum_{j} \alpha_{j} \eta^{(j)} .
$$


The general form for $\eta_{S}$ in a circular domain without islands is given in $\S 3$ and the form of $\eta_{S}$ for an arbirary domain at large Kelvin number is discussed in $\S 4$.

The contribution of the waves to the evolution follows by eliminating $u, v$ from (2.23) to give the inhomogeneous Klein-Gordon equation

$$
\eta_{t t}+K^{2} \eta-\nabla^{2} \eta=K^{2} \eta_{I},
$$

where $t$ has been scaled on $L / c$, the time for a Kelvin wave to cycle the basin. The solution thus consists of the sum of the steady solution $\eta_{S}$, a Kelvin wave, and a superposition of free Poincaré waves satisfying the homogeneous form of (2.36). The form for a circular domain is found as a Laplace transform in $\S 3$.

\section{A circular domain without islands}

For a circular domain of radius $L$ equation (2.36) and boundary condition (2.20) become

$$
\begin{gathered}
\frac{\left(r \eta_{r}\right)_{r}}{r}+\frac{\eta_{\theta \theta}}{r^{2}}-\eta_{t t}-K^{2} \eta=-K^{2} \eta_{I}, \\
r \eta_{r t}+K \eta_{\theta}=0, \quad \text { at } \quad r=1 .
\end{gathered}
$$

for polar co-ordinates $(r, \theta)$. Since the problem is linear, we can make the Fourier azimuthal decomposition

$$
\eta=\sum_{-\infty}^{\infty} \eta_{n} \exp (i n \theta)
$$

Omitting the Fourier subscript $n$, we find that (3.1) reduces to

$$
\frac{\left(r \eta_{r}\right)_{r}}{r}-\frac{n^{2} \eta}{r^{2}}-\eta_{t t}-K^{2} \eta=-K^{2} \eta_{I},
$$

while the boundary condition (3.2) and the initial condition (2.21) reduce to

$$
\begin{gathered}
r \eta_{r t}+i n K \eta=0, \quad \text { at } \quad r=1 . \\
\eta=\eta_{I n}(r), \eta_{t}=0, \quad \text { at } \quad t=0 .
\end{gathered}
$$

\subsection{Free waves}

First, we examine the free wave modes, as in Lamb (1932). Thus we set $\eta_{I}=0$ and seek solutions for which

$$
\eta=F(r) \exp (-i \omega t)
$$

Then the set $(3.4,3.5)$ reduce to

$$
\begin{gathered}
\frac{\left(r F_{r}\right)_{r}}{r}-\frac{n^{2} F}{r^{2}}+\omega^{2} F-K^{2} F=0, \\
\omega r F_{r}-n K F=0, \quad \text { at } \quad r=1 .
\end{gathered}
$$

The solution of (3.8) which is smooth at $r=0$ is

$$
F=J_{n}(\kappa r) \text { where } \kappa^{2}=\omega^{2}-K^{2} .
$$

The boundary condition then gives

$$
\omega \kappa J_{n}^{\prime}(\kappa)-n K J_{n}(\kappa)=0 .
$$

This determines $\omega$ as a function of $K$ and the transverse wavenumber $n$. For each $n$ there are an infinite number of Poincaré wave solutions, all superinertial, that is $\kappa^{2}=$ 
$\omega^{2}-K^{2}>0$. There is also a single subinertial mode where $\kappa^{2}=-\gamma^{2}<0$, and we replace $(3.10,3.11)$ with

$$
\begin{gathered}
\eta=I_{n}(\gamma r) \quad \text { where } \gamma^{2}=K^{2}-\omega^{2} . \\
\omega \gamma I_{n}^{\prime}(\gamma)-n K I_{n}(\gamma)=0, \quad n=1,2, \cdots .
\end{gathered}
$$

The dispersion relation (3.11) can be solved numerically, or graphically as by Lamb (1932). When $n=0$, the only solutions are the superinertial Poincaré waves with $\kappa=j_{s 0}^{\prime}$, the zeros of $J_{0}^{\prime}$, indexed by $s=1,2,3, \cdots$, and so

$$
\omega^{2}=K^{2}+j_{s 0}^{\prime 2} .
$$

For $n \neq 0$ it is sufficient to consider only $n=1,2,3, \cdots$, as the case $n<0$ is recovered by replacing $\omega$ with $-\omega$. Likewise we can take $K>0$ (for the northern hemisphere) without loss of generality, as $K<0$ is also recovered by replacing $\omega$ with $-\omega$. Let

$$
\Phi(\kappa)=\frac{\kappa J_{n}^{\prime}(\kappa)}{n J_{n}(\kappa)}=1-\frac{\kappa J_{n+1}(\kappa)}{n J_{n}(\kappa)},
$$

and the dispersion relation becomes

$$
\frac{K}{\omega}= \pm \frac{K}{\left(K^{2}+\kappa^{2}\right)^{1 / 2}}=\Phi(\kappa) .
$$

Here the alternate signs correspond to $\omega>0,<0$ respectively. Note that when $\kappa \rightarrow$ $0, \Phi(\kappa) \approx 1-\kappa^{2} / 2 n(n+1)$. Otherwise $\Phi \rightarrow \pm \infty$ as $\kappa \rightarrow j_{s n}$, the zeros of $J_{n}(\kappa)$, and $\Phi=0$ at $\kappa=j_{s n}^{\prime}$, the zeros of $J_{n}^{\prime}(\kappa)$, where for each fixed $n, j_{s n}^{\prime}<j_{s n}$. Consequently, $\Phi(\kappa)$ varies from 1 to $-\infty$ in $0<\kappa<j_{1 n}$ passing through zero at $\kappa=j_{1 n}^{\prime}$, then from $\infty$ to $-\infty$ in $j_{1 n}<\kappa<j_{2 n}$, passing through 0 at $\kappa=j_{2 n}^{\prime}$, and so on. There are clearly then an infinite number of intercepts with the left-hand side $K / \omega$, one with $\omega<0$ in each interval $j_{s n}^{\prime}<\kappa<j_{s n}$, and one with $\omega>0$ in each interval $j_{s n}<\kappa<j_{(s+1) n}^{\prime}$. There may also be an intercept for the case $\omega>0$ for $0<\kappa<j_{1 n}^{\prime}$ if $K^{2}<n(n+1)$, which can be interpreted as a Kelvin wave. The subinertial case can be analyzed in a similar manner. Let

$$
\Psi(\gamma)=\frac{\gamma I_{n}^{\prime}(\gamma)}{n I_{n}(\gamma)}=1+\frac{\gamma I_{n+1}(\gamma)}{n I_{n}(\gamma)}
$$

so that the dispersion relation (3.13) becomes

$$
\frac{K}{\omega}= \pm \frac{K}{\left(K^{2}-\gamma^{2}\right)^{1 / 2}}=\Psi(\gamma)
$$

In this case it is readily shown that only the case $\omega>0$ is possible, and then only if $K^{2}>n(n+1)$, since now $\Psi(\gamma)$ increases from 1 to $\infty$ for $0<\gamma<\infty$. A typical set of values is shown in Table 1 , where for each $n$ we index the modes with $s= \pm 1, \pm 2, \pm 3, \cdots$ according to the magnitude of the roots, and the sign is that of $\omega$. In general for each $|s|, \mid \omega$ increases with $n$, and for each $n, \mid \omega$ increases with $|s|$; also, for each fixed $n,|s|$, the negative frequencies are larger in absolute value than the positive frequencies, but converge in absolute value as $|s|$ increases.

In the limit of special interest when $K \rightarrow \infty$, for the super initial waves $\omega$ scales with $K$ and then the superinertial Poincaré waves are given by $\kappa \approx j_{s n}^{\prime}$, the zeros of $J_{n}^{\prime}$, indexed by $s=1,2,3, \cdots$, and so

$$
\omega^{2} \sim K^{2}+j_{s n}^{\prime 2}
$$

Note that as $K \rightarrow \infty$, the frequencies accumulate towards $K$, that is, in dimensional variables towards the inertial frequency $f$. However, for the sub-inertial Kelvin wave, 


\begin{tabular}{ccccccc}
\hline$s$ & +1 & +2 & +3 & -1 & -2 & -3 \\
\hline$n=1$ & 1.51 & 5.39 & 8.58 & -2.35 & -5.46 & -8.61 \\
$n=2$ & 1.93 & 6.59 & 9.91 & -2.80 & -6.64 & -9.92 \\
\hline
\end{tabular}

TABLE 1. Frequencies $\omega$ for $n=1,2$ and $s= \pm 1, \pm 2, \pm 3$ when $K=1$

although $\gamma \approx K$, the frequency remains of order unity, given by, from (3.13),

$$
\omega \sim \frac{n I_{n}(K)}{I_{n}^{\prime}(K)} \sim n, \quad n=1,2, \cdots
$$

This is valid when $K^{2}>n(n+1)$.

\subsection{The initial-value problem and the steady state}

The initial value problem for the $n$th Fourier mode is the set $(3.4,3.5,3.6)$. This can be solved with Laplace transforms. Note that the mass constraint (2.27) is automatically satisfied by all modes $n \neq 0$, and for the mode $n=0$ becomes

$$
\int_{0}^{1} \eta_{0} r d r=\frac{M}{2 \pi}=\int_{0}^{1} \eta_{I 0} r d r
$$

The final solution consists of a sum of free waves and the steady solution, $\eta_{S}(r)$, for each Fourier component, given by

$$
\frac{\left(r \eta_{S r}\right)_{r}}{r}-\frac{n^{2} \eta_{S}}{r^{2}}-K^{2} \eta_{S}=-K^{2} \eta_{I}(r),
$$

with the boundary condition, a simplified form of (2.33),

$$
\begin{aligned}
\eta_{S}=0 \quad \text { at } \quad r=1, \quad \text { when } \quad n \neq 0, \\
\eta_{S r}=0 \quad \text { at } \quad r=1, \quad \text { when } n=0 .
\end{aligned}
$$

Note that the boundary condition (3.17) ensures that $\eta_{S}$ automatically satisfies the mass constraint (3.14) in full. The ordinary differential equation (3.15) with the boundary condition (3.16) can be readily solved in terms of Bessel functions as follows

$$
\begin{aligned}
& \eta_{S}=A_{n} I_{n}(K r)+B_{n} K_{n}(K r) \\
& +K\left\{I_{n}(K r) \int_{r}^{1} K_{n}(K r) \eta_{I} r d r-K_{n}(K r) \int_{r}^{1} I_{n}(K r) \eta_{I} r d r\right\}
\end{aligned}
$$

where the constants $A_{n}, B_{n}$ are determined by the boundary condition (3.16) or (3.17), and the requirement that the solution be bounded as $r \rightarrow 0$. Thus we get that

$$
A_{n} I_{n}(K)+B_{n} K_{n}(K)=\left(0, M_{0}\right) \quad \text { as } \quad n \geq 1, n=0, \quad B_{n}=K \int_{0}^{1} I_{n}(K r) \eta_{I} r d r
$$

where $M_{0}$ is determined by the mass constraint (3.14). Note that in the limit of interest when $K \rightarrow \infty, \eta_{S}$ is order unity. Indeed, except in a boundary layer near $r=1$ of thickness $a=1 / K,(3.15)$ shows that $\eta_{S} \approx \eta_{I}$.

Then to solve the initial value problem, we introduce $\phi(r, t)=\eta-\eta_{S}$, the difference between the full solution and the steady-state solution. In the unbounded linear problem $\phi$ is a transient response that decays at large time due to Poincaré wave radiation. Here, 
in the bounded domain $\phi$ consists of constant amplitude free Poincaré modes and Kelvin waves cycling around the boundary, together satisfying,

$$
\begin{aligned}
\phi_{t t} & =\frac{\left(r \phi_{r}\right)_{r}}{r}-\frac{n^{2} \phi}{r^{2}}-K^{2} \phi, \\
\phi_{r t} & =i n K \phi, \quad \text { on } \quad r=1, \\
\phi & =\phi_{I}=\eta_{I}-\eta_{S}, \quad \phi_{t}=0, \quad \text { at } \quad t=0 .
\end{aligned}
$$

Next we take a Laplace transform

$$
\Phi(r, \sigma)=\int_{0}^{\infty} \exp (-\sigma t) \phi(r, t) d t, \quad \phi(r, t)=\frac{1}{2 \pi i} \int_{C} \exp (\sigma t) \Phi(r, \sigma) d \sigma .
$$

Here $C$ is a contour in $\operatorname{Re}[\sigma]>0$ from $\epsilon-i \infty$ to $\epsilon+i \infty$, where $\epsilon>0$ is chosen so that all singularities of $\Phi$ lie to the left of $C$. Then the equation set (3.20) becomes

$$
\begin{aligned}
\sigma^{2} \Phi & =\frac{\left(r \Phi_{r}\right)_{r}}{r}-\frac{n^{2} \Phi}{r^{2}}-K^{2} \Phi+\sigma \phi_{I}, \\
\sigma \Phi_{r} & =i n K \Phi+\phi_{I r}, \quad \text { on } \quad r=1,
\end{aligned}
$$

This ordinary differential equation boundary value problem can now be solved explicitly,

$$
\begin{aligned}
& \Phi=C_{n} I_{n}(\gamma r)+D_{n} K_{n}(\gamma r) \\
& -\frac{\sigma}{\gamma}\left\{I_{n}(\gamma r) \int_{r}^{1} K_{n}(\gamma r) \phi_{I} r d r-K_{n}(\gamma r) \int_{r}^{1} I_{n}(\gamma r) \phi_{I} r d r\right\},
\end{aligned}
$$

where $\gamma^{2}=\sigma^{2}+K^{2}$ and the constants $C_{n}, D_{n}$ are determined by the boundary conditions,

$$
\begin{aligned}
& C_{n}\left[\sigma \gamma I_{n}^{\prime}(\gamma)-i n K I_{n}(\gamma)\right]+D_{n}\left[\sigma \gamma K_{n}^{\prime}(\gamma)-i n K K_{n}(\gamma)\right]=\phi_{I r}(1), \\
& D_{n}=-\frac{\sigma}{\gamma} \int_{0}^{1} I_{n}(\gamma r) \phi_{I r} r d r .
\end{aligned}
$$

As a function of $\sigma, \Phi(\sigma, r)$ has poles at $\sigma=-i \omega$ where

$$
\omega \gamma I_{n}^{\prime}(\gamma)+n K I_{n}(\gamma)=0, \quad \gamma^{2}=K^{2}-\omega^{2} .
$$

The frequencies so determined are precisely those of the free waves determined in section 2.1, see (3.11) and (3.13) where $\gamma=i \kappa, \kappa^{2}=\omega^{2}-K^{2}$ in the former case. In the limit of interest when $K \rightarrow \infty$, the Poincaré waves have high frequencies $\omega$ scaling with $K$ (as in $\S 2.1$ ) and have amplitudes of order $a=1 / K$, most readily inferred directly from (3.22). On the other hand in this same limit, the Kelvin wave has an order unity frequency, an order unity amplitude, and is confined to the outer boundary within a layer of order a. Hence the complete solution then consists essentially of simply the steady solution, adjusted at the boundary by a Kelvin wave.

\subsection{Numerical results}

Although the expressions $(3.21,3.23)$ formally provide an explicit solution for $\phi$, in general the integrals involving $\phi_{I}$ are quite difficult to evaluate: it is simpler to solve (3.20) directly by high-accuracy spectral discretisation. Here (3.20) is discretised with 128 Chebyshev points over $-1 \leq x \leq 1$, following Fornberg (1995), and using an appropriate parity condition to extend the solution to negative $r$. This extension avoids stringent CFL conditions at the origin and automatically copes with the polar co-ordinate singularity there. The spatially discretised equations were integrated forward in time with an AdamsBashforth method. Integrations at higher resolutions differed by less than $10^{-8}$ from 


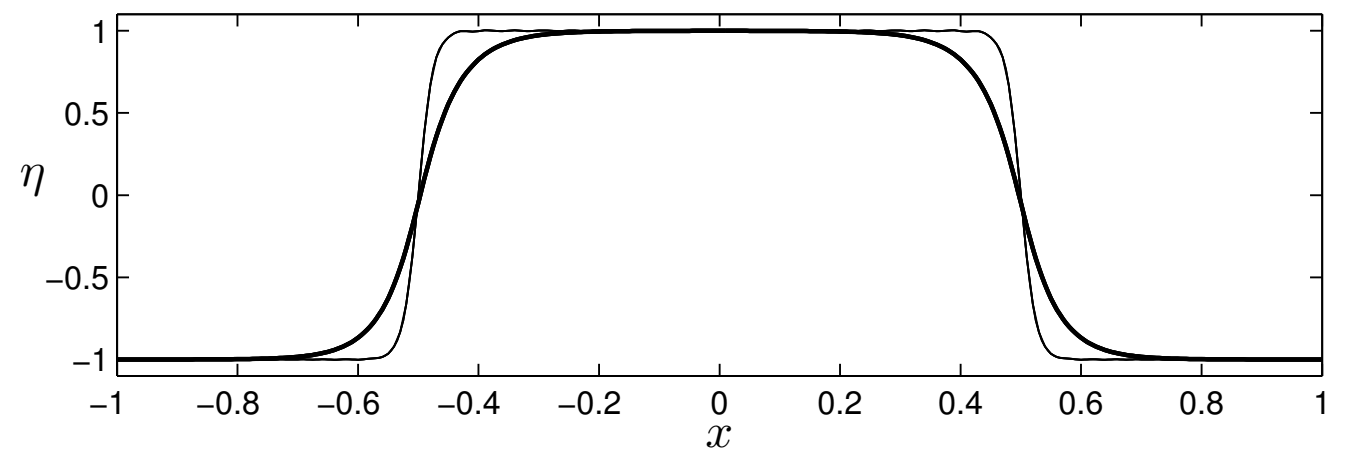

FiguRE 2. A cross-section along the line $y=0$ of the initial surface perturbation $\eta_{I 0}$ for an axisymmetric $(n=0)$ top-hat profile (thin line) and the steady adjusted solution $\eta_{S}$ (bold line). Here $a=0.05$ so the Rossby radius is one-twentieth the basin radius. The steep initial step has been smoothed by a layer of thickness of order $a$.

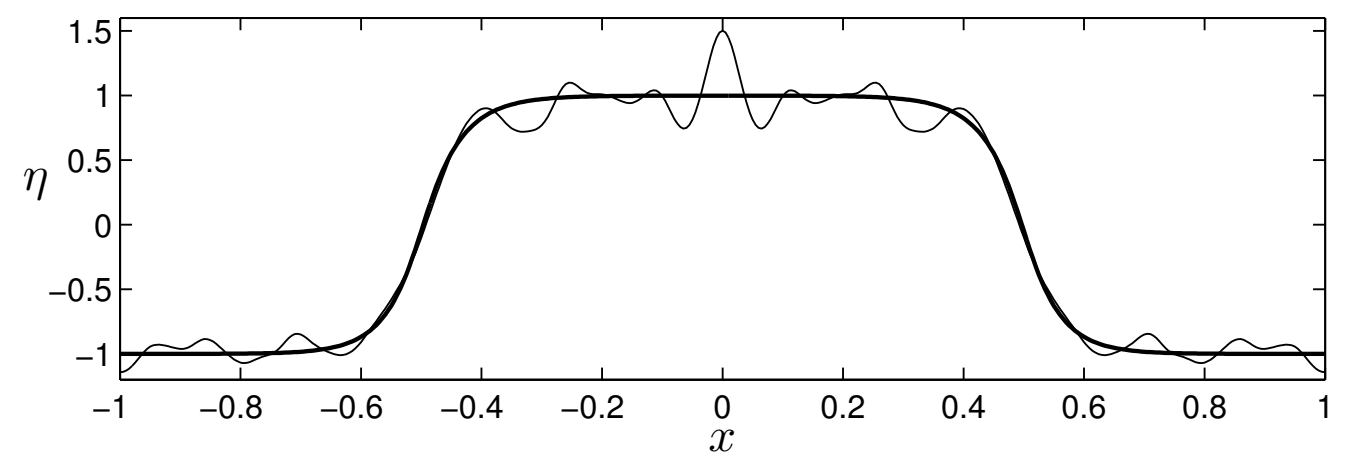

Figure 3. A snapshot at $t=10$ along the line $y=0$ of the surface perturbation $\eta$ (thin line) for the top-hat profile of Figure 2. As in 2, the bold line gives the steady adjusted solution $\eta_{S}$. There is no Kelvin wave in the evolution and the Poincaré waves have amplitudes, wavelengths and non-dimensional periods of order $a$. The average surface displacement over the interval $0 \leq t \leq 10$ is graphically indistinguishable from $\eta_{S}$.

those presented here. For small $K$ (large $a$ ) when the Rossby radius is large compared to the basin diameter, the rotational effects are weak and the flow evolves similarly to non-rotating flow. Hence, the results presented here are for $a=0.05, K=20$, when the Rossby radius is one twentieth the basin radius, the non-dimensional inertial period is approximately $\pi / 10$, and the Kelvin wave cycles the basin in a non-dimensional time of approximately $2 \pi$.

\subsubsection{Case 1. Axisymmetric flow: $n=0$.}

Consider the top-hat initial condition

$$
\eta_{I 0}=-\tanh \left[\left(r-r_{0}\right) / r_{w}\right],
$$

for a downward step of height 2 centred at radius $r_{0}$ (taken as 0.5 here and below) and of width $r_{w}$ (taken as 0.025 here and below). Figure 2 shows this initial condition and the steady adjusted solution $\eta_{S}$ where the steep initial step has been smoothed by a layer of thickness of order $a=1 / K$.

Figure 3 shows a snapshot at $t=10$ of the surface perturbation $\eta$ for this initial profile. As the flow is axisymmetric no Kelvin wave is excited in the evolution and, for the small value of the Rossby radius $a$ here, the Poincaré waves have amplitudes, wavelengths and 


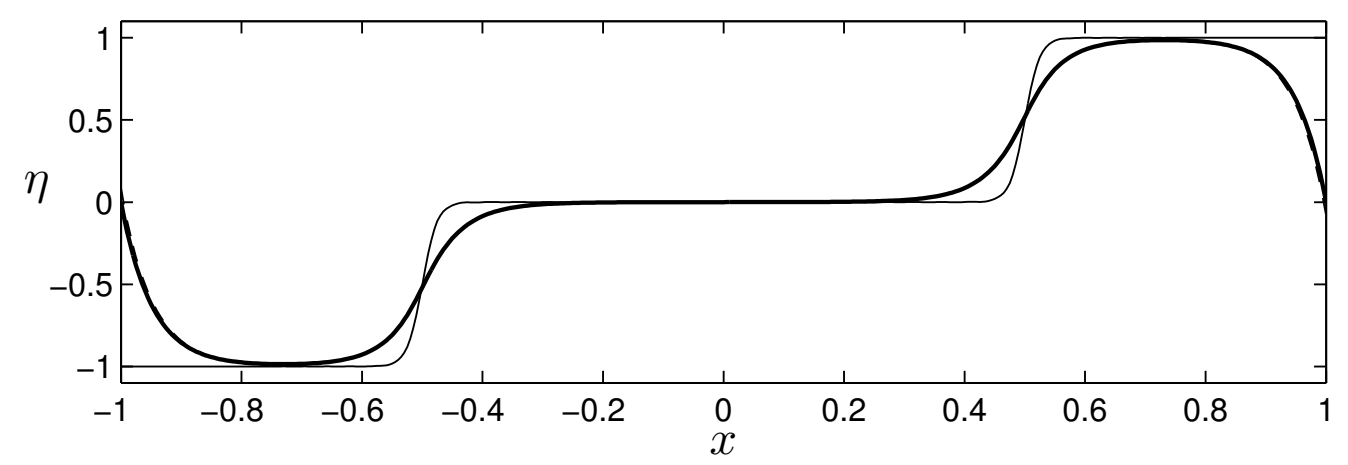

Figure 4. A cross-section along the line $y=0$ of the initial surface perturbation $\eta_{I 1}$ for an azimuth one $(n=1)$ profile (thin line) and the steady adjusted solution $\eta_{S}$ (bold line). As in Figure 2, $a=0.05$ so the Rossby radius is one-twentieth the basin radius. The steep initial step has been smoothed by layers of thickness of order $a$. A second layer of thickness $a$ at the boundary adjusts the interior steady solution to the zero-displacement condition at the wall.

non-dimensional periods of order $a$. The average surface displacement over the interval $0 \leq t \leq 10$ is graphically indistinguishable from $\eta_{S}$. The effect of the Poincaré waves averages to zero over sufficiently many inertial periods.

\subsubsection{Case 2. Non-axisymmetric single-azimuth flow: $n=1$.}

Case 2a. A stepped profile

Consider azimuthal dependence $n=1$, with the initial radial profile

$$
\eta_{I 1}=0.5\left\{1+\tanh \left[\left(r-r_{0}\right) / r_{w}\right]\right\}
$$

giving an upward step of height 1 (at $r=r_{0}$ and of width $r_{w}$, as above). Figure 4 shows this initial condition and the steady adjusted solution $\eta_{S}$ where the steep initial step has been smoothed by layers of thickness of order $a$ and a second layer of thickness $a$ at the boundary adjusts the interior steady solution to the zero-displacement condition at the wall, effectively the condition that there can be no tangential gradient in the steady solution at a solid boundary and consequently no normal component of velocity there. Figure 5 shows a snapshot at $t=10$ of the surface perturbation $\eta$ for this initial profile. Here the flow is not axisymmetric so a single Kelvin wave mode is excited in the evolution. The wave has amplitude of order the difference between the initial and steady states at the wall, period of order $2 \pi$ and is confined to with a distance of order the Rossby radius of the boundary. This can be simply modelled by adding the appropriate Kelvin wave mode of this amplitude to the steady solution as shown by the dashed line in Figure 5. For the small value of the Rossby radius $a$ here it is sufficient to use the asymptotic expression for the Bessel function to give the simple Kelvin wave term

$$
\eta_{K}=\eta_{I 1}(1) \exp [-K(1-r)] \cos (\theta-t) \text {. }
$$

Again, for the small value of the Rossby radius $a$ here, the Poincaré waves have amplitudes, wavelengths and non-dimensional periods of order $a$, making up the small difference between the dashed and solid lines in Figure 5. The average surface displacement over intervals long compared to the inertial period $2 \pi / a$ but short or comparable to the Kelvin wave period $2 \pi$ is indistinguishable from the combined steady and Kelvin wave solution with the average over times long compared to the Kelvin wave period approaching $\eta_{S}$ 

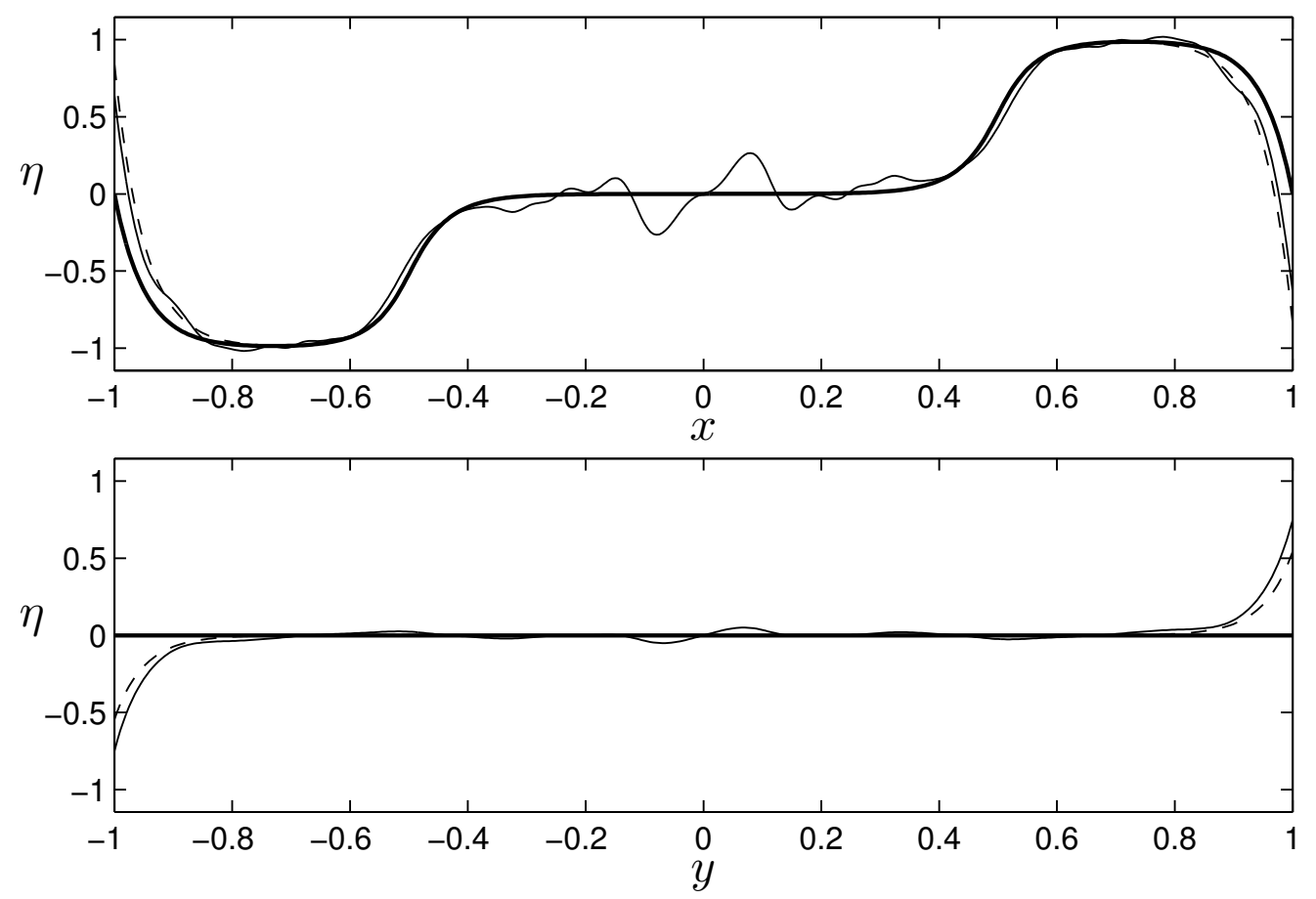

Figure 5. Snapshots at $t=10$ along the lines $y=0$ (upper) and $x=0$ (lower) of the surface perturbation $\eta$ (thin line) for the $n=1$ profile of Figure 4. As in Figure 4, the bold line gives the steady adjusted solution $\eta_{S}$. The dashed line gives the sum of the steady solution and the single Kelvin wave mode, with period of order $2 \pi$ and confined to within a distance of order the Rossby radius of the boundary. The Poincaré waves have amplitudes, wavelengths and non-dimensional periods of order $a$.

\section{Case 2b. A uniformly sloping profile: closed form solution}

For azimuthal dependence $n=1$ with initial radial profile, giving a uniformly sloping initial surface displacement,

$$
\eta_{I 1}=r
$$

the interior flow is already in geostrophic balance and the solution can be obtained explicitly without requiring the steady solution. Writing $\psi(r, t)=\eta(r, t)-r$ and introducing the Laplace transform, $\Psi(r, \sigma)$, so

$$
\begin{aligned}
& \frac{\left(r \Psi_{r}\right)_{r}}{r}-\frac{\Psi}{r^{2}}-\left(\sigma^{2}+K^{2}\right) \Psi=0, \\
& \sigma \Psi_{r}+i K \Psi=i K \quad \text { on } \quad r=1,
\end{aligned}
$$

gives

$$
\Psi(r, \sigma)=\frac{i K I_{1}(\gamma r)}{\sigma \gamma I_{1}^{\prime}(\gamma)+i K I_{1}(\gamma)}=\frac{i K I_{1}(\gamma r)}{\sigma \gamma I_{0}^{\prime}(\gamma)+(i K-\sigma) I_{1}(\gamma)},
$$

where $\gamma=\left(\sigma^{2}+K^{2}\right)^{1 / 2}$ and we have used the relation $\gamma I_{1}^{\prime}(\gamma)=\gamma I_{0}(\gamma)-I_{1}(\gamma)$. Summing the residues at the zeros of the denominator in the Bromwich integral gives $\psi(r, t)$. In the limit of large $K$ (small $a$ ) only the residue corresponding to the Kelvin wave remains non-zero, giving the result (3.27), where here $\eta_{I}(1)=1$. 


\subsection{The large $K$ limit}

Example $2 \mathrm{~b}$ shows that in the circular domain in the small $a$, large $K=1 / a$ limit the leading order (in $a$ ) response for any $n$ consists of the steady response plus a mode $n$ Kelvin wave. For more general initial conditions the leading order response consists of the steady state response plus a superposition of boundary-confined Kelvin waves. Moreover, the average over time periods long compared to the Poincaré waves periods is closely estimated by this combined response. Now the uniformly first-order (in $a$ ) steady solution satisfies equation (2.25) subject to the boundary conditions (2.26) as noted in $\S 2$ and since $a$ is small, the form of the steady solution follows straightforwardly. Away from the boundary, and outside regions where the initial elevation varies on scales of order $a$ or less,

$$
\eta_{S}=\eta_{I}
$$

and the initial elevation is unchanged. Rapidly-varying regions of $\eta_{0}$ are smoothed by layers of thickness $a$, and a similar layer of thickness $a$ can be present around the boundary to satisfy the circulation constraint. The remainder of the response is the Kelvin wave response, confined to within a distance $a$ from the boundary. Thus we introduce the scaled normal co-ordinate $\xi=K(1-r) \geq 0$. Then the leading order Kelvin wave response, $\eta_{K}(\xi, \theta, t)$ satisfies

$$
\eta_{K \xi \xi}-\eta_{K}=0, \quad \xi>0,
$$

subject to

$$
\begin{aligned}
\eta_{K \xi t}-\eta_{K \theta} & =0, \quad \text { on } \quad \xi=0, \quad t>0, \\
\eta_{K}(1, \theta)+\eta_{S}(1, \theta) & =\eta_{I}(1, \theta), \quad \text { on } \xi=0, \quad t=0 .
\end{aligned}
$$

The solution of (3.32), (3.33) is

$$
\eta_{K}(\xi, \theta, t)=F(\theta+t) \exp (-\xi),
$$

where

$$
F(\theta)=\eta_{I}(1, \theta)-\eta_{S}(1, \theta)=\eta_{I}(1, \theta)-\alpha_{0} .
$$

The departure of the initial surface displacement at the wall from its long term average $\left(\alpha_{0}\right)$ propagates at the constant dimensional longwave speed $c$ around the wall (with the wall to the right, as expected) without change of form.

\section{Adjustment in arbitrary domains for large $K$}

The generalisation of the results of $\S 3$ for large $K$ to the arbitrary basin with islands of $\S 2$ follows immediately. Provided the boundary geometry is slowly-varying on the (small) scale of the Rossby radius, so Kelvin wave energy is not scattered into Poincaré waves, the leading order (in $a$ ) flow can be expressed as the sum of steady, $\eta_{S}$, and Kelvin wave, $\eta_{K}$, components where the steady component satisfies (2.25) throughout $\mathcal{D}$ subject to the boundary conditions (2.26). The Kelvin wave component satisfies $(3.32)$ at each $\partial \mathcal{D}_{i}$ provided $\xi$ is generalised to be the normal distance, scaled on $a$, measured inwards from the relevant boundary. The boundary condions at each $\partial \mathcal{D}_{i}$ become

$$
\begin{aligned}
\eta_{K \xi t}^{(i)}+\eta_{K s}^{(i)} & =0, \quad \text { on } \partial \mathcal{D}_{i}, \quad t>0, \\
\eta_{K}^{(i)} & =\eta_{I}-\alpha_{i}=k_{i}(s)(\text { say }), \quad \text { on } \partial \mathcal{D}_{i}, \quad t=0
\end{aligned}
$$




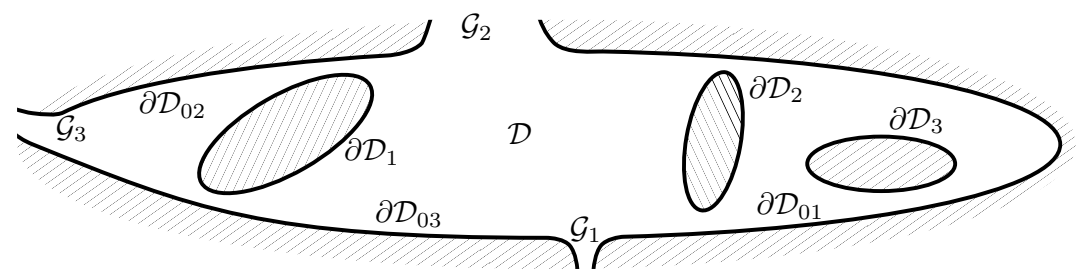

Figure 6 . The basin $\mathcal{D}$ with islands with boundaries $\partial \mathcal{D}_{i}(i=1,2, \ldots)$ and outer boundary $\partial \mathcal{D}_{0}$ split into sections $\partial \mathcal{D}_{0 j}(j=1,2, \ldots)$ by gaps $\mathcal{G}_{j}$.

where $s$ is the arc length (measured anti-clockwise around $\partial \mathcal{D}_{0}$ and clockwise around $\left.\partial \mathcal{D}_{i}, i=1,2, \ldots\right)$. The solution $\eta_{K}^{(i)}$ is thus

$$
\eta_{K}^{(i)}(\xi, s, t)=k_{i}(s-t) \exp (-\xi),
$$

with, again, the departure of the initial surface displacement on each $\partial \mathcal{D}$ from its long term average $\left(\alpha_{i}\right)$ propagating around the boundary at (dimensional) speed $c$ with the wall to the right and without change of form.

In practice basins may not be completely closed and small gaps may be present in the outer boundary. This certainly applies to the Arctic ocean, but is also usually the case even for large lakes. Figure 6 shows a typical basin with gaps $\mathcal{G}_{j},(j=1,2, \ldots)$ breaking the outer boundary $\partial \mathcal{D}_{0}$ into sections $\partial \mathcal{D}_{0 j}$ (with $\partial \mathcal{D}_{0 j}$ extending clockwise from $\mathcal{G}_{j}$ ). The analysis above extends directly provided allowance is made for the absence of a circulation condition on $\partial \mathcal{D}_{0}$. If all gaps are wider than the relevant Rossby radius then the steady elevation $\alpha_{0 j}$ on $\partial \mathcal{D}_{0 j}$ is given simply by the upstream elevation of the corresponding boundary of gap $\mathcal{G}_{j}$, and the steady component $\eta_{S}$ satisfies (2.25) subject to the boundary conditions (2.26), but taking the values $\alpha_{0 j}$ on each section $\partial \mathcal{D}_{0 j}$. The Kelvin wave on each section is driven, as above, by the difference between the initial elevation and the average $\alpha_{0 j}$ but, for the broken outer boundary, rapidly propagates out of the flow domain. If any gap $\mathcal{G}_{j}$ is narrower than the Rossby radius then the determination of the constant steady elevation on $\partial \mathcal{D}_{0 j}$ is unchanged if $\alpha_{0 j-1}=\alpha_{0 j}$ (which is so if the adjustment occurs in the basin rather than between gaps with different initial surface elevations) so there is no incompatibility in the steady elevations on either boundary of $\mathcal{G}_{j}$, but otherwise depends on the details of the gap geometry.

\section{Discussion}

Our main conclusion is that for baroclinic vertical modes when the non-dimensional Rossby radius $a$ is small the boundary has little effect on the interior solution. Indeed, apart from the presence of small-amplitude $O(a)$ Poincaré waves which are trapped, conservation of potential vorticity implies that the steady interior solution for the height field is hardly changed from the initial state. Since this applies to each vertical mode, if the Rossby radii of the modes are small (in fact it is sufficient for the Rossby radius of the lowest vertical mode present to be small) and interior potential vorticity smooth then it is unnecessary to separate the potential vorticity into vertical modes to follow the interior adjustment. Of course, this final interior steady potential vorticity distribution, being in geostrophic balance, will be accompanied by a geostrophic velocity field generated during the adjustment process, but this velocity follows immediately from the usual geostrophic relations for the horizontal velocities. Near the boundary a superposition of Kelvin waves decaying towards the interior over distances of order the Rossby radius carry the initial surface displacement around the boundary without change of form. 
Although our detailed analysis has been for a circular domain of constant depth, in the small $O(a)$ limit, the results extend readily to domains of arbitrary shape containing islands whether the basins are completely closed or with outer boundaries broken by gaps. In this case, we suggest that during adjustment, such gaps and islands have a large (order unity) local effect but that for large $K$ this is confined to an unsteady region of width $a=1 / K$ close to the gaps and islands.

Two unrelated nonlinear effects can appear in the evolution. Firstly, with larger initial displacements the velocities engendered can be sufficiently large that the advection of potential vorticity can be important. Secondly, the Kelvin wave disturbance propagating around the boundary can break even at small amplitude if the initial tangential gradients are sufficiently large. These effects will be examined in more detail elsewhere.

\section{REFERENCES}

FornberG, B. 1995 A pseudospectral approach for polar and spherical geometries. SIAM J. Sci. Computing 16, 1071-1081.

Gill, A. 1982 Atmosphere-Ocean Dynamics. Academic Press.

Gill, A. E. 1976 Adjustment under gravity in a rotating channel. J. Fluid Mech. 77, 603-621.

Gomez-Giraldo, A., Imberger, J. \& Antenucci, J. P. 2006 Spatial structure of the dominant basin-scale internal waves in lake kinneret. Limnology and Oceanography 51, 229-246.

Johnson, E. R. 1989 Scattering of shelf waves by islands. J. Phys. Oceanogr. 19, 1311-1316.

LAMB, H. 1932 Hydrodynamics. CUP.

LiN, C. C. 1941 On the motion of vortices in two-dimensions. I. Existence of the Kirchhoff-Routh function. Proc. Nat. Acad. Sci. 27, 570-577.

Luneva, M. V., Willmott, A. J. \& Morales, M. A. 2012 Geostrophic adjustment problems in a polar basin. Atmosphere-Ocean 50, 134-155.

Rhines, P. B. 1969 Slow oscillations in an ocean of varying depth. 2. Islands and seamounts. J. Fluid Mech. 37, 191- 205.

Stocker, R. \& Imberger, J. 2003 Energy partitioning and horizontal dispersion in the surface layer of a stratified lake. J. Phys. Ocean. 33, 512-529.

Wake, G. W., Hopfinger, E. J. \& Ivey, G.N. 2007 Experimental study on resonantly forced interfacial waves in a stratified circular cylindrical basin. J. Fluid Mech. 582, 203-222.

Wake, G. W., Ivey, G.N., Imberger, J. \& McDonald, N. R. 2005 The temporal evolution of a geostrophic flow in a rotating stratified basin. Dyn. Atmos. Oceans 39, 189-210.

Wake, G. W., Ivey, G.N., Imberger, J., McDonald, N. R. \& Stocker, R. 2004 Baroclinic adjustment in a rotating circular basin. J. Fluid Mech. 515, 63-86. 